\section{Síndrome del intestino permeable: una diana terapéutica en el control de la inflamación sistémica de origen autoinmune a tener en cuenta}

\section{Señor director}

La última mitad del siglo XX ha supuesto una revolución en el campo de la medicina, evidente para cualquier clínico; el descubrimiento de los antibióticos, los avances quirúrgicos, la endoscopia y las técnicas de imagen permiten alcanzar objetivos terapéuticos con los que los médicos antiguos soñaban. Además, en los últimos años estamos asistiendo a un gran avance debido a las terapias basadas en el uso de anticuerpos monoclonales para el control de numerosas patologías mediadas por desórdenes del sistema inmune. No obstante, es evidente que faltan hipótesis etiológicas susceptibles de ser tratadas para este grupo tan amplio de enfermedades.

En el siglo $V$ a.c. se postulaba que la adecuada nutrición era la una de las piedras angulares para el mantenimiento de la salud; Hipócrates ya escribía "las investigaciones acerca del régimen alimenticio son una de las cuestiones más importantes y dignas de merecer nuestra atención" [1].

La relación entre los trastornos intestinales y las enfermedades sistémicas inflamatorias es bien conocida desde hace décadas $[2,3,4]$ pero no existen abordajes de tratamiento adecuadamente protocolizados. Es conocida la mayor incidencia de síntomas articulares en los enfermos afectados de Enfermedad Inflamatoria Intestinal [5].

El intestino delgado juega un papel indispensable en la homeostasis; es la principal fuente de contacto del organismo con el medio externo y se somete diariamente a una carga de nutrientes y sustancias extrañas, potencialmente dañinas; este papel defensivo se apo-
Luis Diaz Vidal', Mario Sánchez Pérez², Yolanda Aranda García 3

1 Especialista en Medicina Interna Hospital Universitario Ramón y Cajal

2 Especialista en Medicina Familiar y Comunitaria Hospital Universitario Ramón y Cajal

3 Especialista en Medicina Familiar y Comunitaria Hospital Universitario Ramón y Cajal

Correspondencia:

Dr. L. Diaz Vidal

झ luisdvidal@gmail.com 
ya por la presencia de células inmunes que residen en él, representando hasta el $70 \%$ de la totalidad del tejido inmune sistémico (6). La base tisular y funcional de esta función la determina la barrera intestinal y está formada por los enterocitos con sus uniones intercelulares, la lámina propia y el tejido linfoide asociado. En condiciones fisiológicas esta barrera impide mediante una permeabilidad selectiva el paso de múltiples sustancias intraluminales al torrente circulatorio. La integridad de ésta puede alterarse debido a la ingestión de sustancias con alto poder inmunógeno, la alteración de la microflora intestinal o la presencia de elementos esencialmente lesivos para la mucosa como los AINE, por ejemplo [7].

El mecanismo mediante el cual se produce la permeabilidad intestinal es desconocido, pero hay evidencias que involucran al sistema inmunitario, la flora intestinal y la dieta, fundamentalmente.

El intestino delgado humano contiene un elevado número de bacterias comensales, esenciales para una adecuada homeostasis. Estos microorganismos colaboran a su vez en la integridad de la barrera intestinal [8].

La composición de la flora intestinal en casos de enfermedad inflamatoria de origen reumático (artritis reumatoide, espondilitis anquilosante) está bien documentado, con una proliferación excesiva de especies de klebsiella, Proteus y E.coli $[9,10,11]$. Otro trabajo arroja que existe una población bacteriana distinta en individuos vegetarianos [12].

Modelos experimentales ponen de manifiesto la presencia de anticuerpos dirigidos contra los lipopolisacáridos de bacterias gram negativas así como un aumento de proteasas intestinales aisladas en la materia fecal [13]. Estudios in Vitro involucran a citoquinas como la IL 13, TNF e IFN gamma elaborados por las células inmunes, así como radica- les libres como el oxido nítrico pueden producir permeabilidad intestinal [14]. La presencia de moléculas reconocidas como extrañas por el sistema inmunitario intestinal desencadenaría una cascada inflamatoria con la producción de citoquinas y radicales libres que contribuirían a producir y perpetuar el daño en la integridad de la barrera [15].

Recientemente ha surgido el concepto del Síndrome del Intestino Permeable (SIP), en el cual se produciría una disrupción física y funcional de la barrera intestinal a consecuencia de la cual, sustancias con alto poder inmunógeno (residuos bacterianos y alimentarios) accederían a la circulación sistémica; ésto desencadena una cascada de mediadores inflamatorios, relacionados con el desarrollo de enfermedades sistémicas; estas sustancias procederían de la dieta y de residuos y antígenos bacterianos, fundamentalmente [16].

Existen varios modelos experimentales en los que se demuestra el paso intestinal de sustancias inertes como el manitol y el polietilenglicol, posteriormente detectadas en la orina en enfermos con enfermedades inflamatorias [17].

\section{Papel de la dieta en el SIP}

La dieta del ser humano ha cambiado drásticamente desde sus orígenes de cazador y recolector. Los estudios fecales arrojan que estos homínidos comían alimentos crudos. En el período neolítico se introdujeron de forma progresiva el sedentarismo, la agricultura y la ganadería con una mayor disponibilidad de alimento [18].

Este drástico cambio en los hábitos alimentarios se ha materializado particularmente en occidente, donde se ha demostrado una mayor incidencia y prevalencia de enfermedad inflamatoria intestinal [19]. 
Pero el verdadero cambio se ha producido en la segunda mitad del siglo XX, con la introducción y posterior generalización de la industria alimentaria. Existen importantes evidencias de que la dieta y el estilo de vida occidental moderno promueve la obesidad, inflamación, la génesis y el empeoramiento de enfermedades inflamatorias sistémicas tales como la artritis reumatoide y el lupus eritematoso sistémico [20,21].

A continuación se detallan los aspectos que distinguen la dieta actual de la primitiva:

\section{El consumo y agricultura intensiva de los cereales}

Las técnicas de hibridación y la agricultura intensiva han modificado profundamente la estructura genética de este cereal; el más consumido en nuestro medio, el trigo candeal es hexaploide, con 21 pares de cromosomas mientras que el trigo ancestral tiene 7 pares de cromosomas. La composición antigénica de ambos es, por tanto, diferente [22]. Estas nuevas proteínas procedentes de los granos de cereal dañan la barrera intestinal al haber sobrepasado éstas la evolución natural de los sistemas enzimáticos de procesamiento de las mismas.

La relación entre el consumo de cereales y las enfermedades de perfil inflamatorio está bien referenciada y es habitual la mejoría de numerosos cuadros mórbidos al cesar su consumo.

Así, Seignalet diseñó un estudio con 200 enfermos afectados de artritis reumatoide. Se obtuvo una desaparición de los síntomas inflamatorios articulares y una mejora en la calidad de vida con dieta en un $80 \%$ de los sujetos. Unos resultados similares se obtuvieron con una cohorte de 100 enfermos con espondilitis anquilosante, en los que se obtuvo una mejoría sostenida de la sintomatología inflamatoria axial en el $96 \%$ de los enfermos que cumplieron adecuadamente la dieta $[23,24]$.
Por otra parte Häfstrom et al demostró una disminución en la inmunoreactividad a antígenos alimentarios en enfermos con AR sometidos a dieta vegetariana [25].

\section{El consumo de leche animal y sus derivados}

Otra de las características de la dieta moderna occidental es la ingesta de productos lácteos, tanto en forma natural como preparada.

La leche de vaca está naturalmente diseñada para satisfacer las necesidades de los terneros, pero no de los humanos; de hecho somos de las pocas especies animales que nos alimentamos con leche de otras especies.

Aunque no hay muchos estudios que estudien la intervención aislada de la retirada de la leche, una estudio puso de manifiesto la reproducción de síntomas intestinales tipo colon irritable en enfermos diagnosticados de Sjögren primario cuando se les introdujeron proteínas de leche vía rectal [26] Otros pequeño estudio evidencian la mejoría en síntomas asmáticos y con migraña después de la retirada de la misma [27].

\section{La cocción de numerosas sustancias}

El uso del calor para cambiar la consistencia y el aspecto de los alimentos es utilizada de forma generalizada. Las elevadas temperaturas cambian la disposición de las proteínas que contienen, dotándoles de un nuevo poder inmunógeno; éstas afectan a la modificación de su estructura espacial, provoca interacciones entre proteínas y en especial, las reacciones de Maillard, mediante las cuales se forman sustancias complejas por la reacción entre los grupos amino de las proteínas y el grupo carbonilo de los azúcares [28,29]. 


\section{El uso de aceites refinados y preparados}

La extracción industrial de aceites se realiza a alta temperatura y con añadidos químicos, lo que aumenta el rendimiento de la extracción. Este proceso provoca el aumento de la proporción de ácidos grasos saturados y la transformación de una fracción de ácidos grasos insaturados trans a la forma cis relacionados con la inflamación sistémica y los eventos cardiovasculares [24].

\section{La contaminación alimentaria}

Los alimentos procesados de forma industrial contienen una cantidad diferente de aditivos químicos como emulgentes, colorantes, espesantes, conservantes. El elevado consumo de éstos sobrecarga la capacidad depurativa y de filtro de la barrera intestinal [30].

\section{La carencia de oligoelementos y minerales}

La agricultura y ganadería intensivas priorizan la velocidad de producción a la calidad de la misma; el producto final carece frecuentemente de oligoelementos, en especial magnesio y vitaminas [31-32].

\section{Recomendaciones}

Seignalet propone una serie de recomendaciones [24] con el fin de conseguir una dieta mínimamente inmunogénica basadas en el reconocimiento de las diferencias antes expuestas, que pueden resumirse en los siguientes puntos:

1. Los cereales quedan prohibidos a excepción del arroz y el trigo sarraceno.

2. No tomar lácteos de ningún tipo. Se recomiendan las leches vegetales (Arroz, soja, Almendras)

3. Cocinar los alimentos a baja temperatura y tomarlos crudos en la medida de lo posible.
4. Utilizar aceites vegetales con la extracción en frío.

5. Consumir alimentos de agricultura y ganadería biológicos.

6. Suplementación de vitaminas y oligoelementos, en especial en magnesio.

7. Suplementación de lactobacilos durante al menos tres meses.

\section{Papel de los AINES en la barrera intestinal}

Los antiinflamatorios no esteroideos son ampliamente utilizados en reumatología. La relación de éstos con la producción de ulceraciones mucosas en el intestino está bien documentada [33,34]. La inhibición de la síntesis de prostaglandinas a nivel mucoso es el desencadenante fisiopatológico fundamental, produciendo una disrupción anatomofuncional de la barrera intestinal, perpetuando así un estado proinflamatorio.

A la vista de los argumentos expuestos creemos necesario aumentar los esfuerzos de investigación, dirigiéndolos a la permeabilidad intestinal mediada por procesos inmunomediados como potencial agente contribuidor a la génesis, o al menos, al mantenimiento de la inflamación sistémica y sus consecuencias.

El precio de esta intervención no parece ser alto, si lo comparamos con los costes de los nuevos tratamientos con anticuerpos monoclonales. Por otro lado, la inclusión de estas medidas podría ayudar reducir la carga socio-económica de enfermedades sistémicas autoinmunes. No obstante ello, harían falta más estudios para evaluar el impacto personal, económico, así como la evolución clínica, radiológica y analítica de estos enfermos para esclarecer el alcance real de una intervención dietética. 


\section{Referencias}

1. Hipócrates, García Gual C, Lara Navas MD, López Férez JA, Cabellos Alvarez B. Tratados hipocráticos. II. Madrid: Gredos; 1983.

2. Anders HJ, AndersenK. The intestinal microbiota, a Leaky gut, and abnormal immunity in kidney disease. Kidney Int. 2013;83(6):1010-6

3. Vighi G, Marcucci F. Allergy and the gastrointestinal system. Clinical and experimental inmunology. 2008;153(suppl 1):3-6.

4. Krack A, Sharma R. The importante of the gastrointestinal system in the patogénesis of heart failure. Eur Heart J. 2005;26(22):2368-74.

5. Shiwashankar et al.Incidence of spondiloarthropaty in patients with ulcerative colitis: a population based study. J Rheumatol. 2013;40(7):1153-7.

6. De Meo MT, Mutlu El, Keshavarzian A. Intestinal permeation and gastrointestinal disease. J Clin Gastroenterol 2002;34:385-96.

7. Oddenwald M, Turner J. Intestinal permeabiliaty defects: is it time to treat? Clinical gastroenterology and hepatology 2013;11:1075-1083.

8. Venkatesh M, Mukherjee S. Symbiotic bacterial metabolites regulate gastrointestinla barrier function via the xenobiótico sensor PXR ant Toll-Like receptor 4. Inmunity 2014;;23. Pii:S1074-7613(14)

9. Newkirk MM, Zbar A. Distinc bacterial colonization patterns of E. Coli subtypes asóciate with rheumatoid factor status in early inflammatory artritis. Rheumatology 2010;49(7)1311-6.

10. Tiwana $H$, Wilson $C$. Antibody responses to gut bacteria in ankilosing spondylitis, rheumatoid artritis, Crohn's disease and ulcerative colitis. Rheumatol Int. 1997;17(1):11-6.

11. Tany $Y$, Tiwana $H$. Antibodies to klebsiella, Proteus and HLA B-27 peptides in japanese patients with ankilosisg spondylitis and rheumatoid artritis. J Rheumatol 1997;24(1):109-14.
12. Toivanen P.A vegan diet changes the intestinal flora. Rheumatology 2002;41:950-951.

13. Carrol I, Maharshak N. Enteric bacterial proteases in inflamatory bowel disease: pathophisiology and clinical implications. 2013; 19(43):7531-7543.

14. Clayburgh DR el al. A porous defense:the Leaky epithelial barrier in intestinal disease. Lab Invest 2004; 84(3):282-91.

15. Fasano A. Et al. Mechanisms of disease: the role of intestinal barrier function in the patogénesis of gastrointestinal autoinmune diseases. Nat clin Pract gastr 2005. 2:416-422.

16. Shen L, Su L. Mechanisms and functional implications of intestinal barrier defects. Dig Dis. 2009;27(4):443-9.

17.Cox MA, Lewis KO. Measurement of smal intestinal permeability markers, lactulose and manitol in serum: results in celiac disease. Dig Dis Sci. 1999;44(2):402-6.

18. Salás J, García Lorda P, Sánchez J. La alimentación y la nutrición a través de la historia. Ed Glosa. Barcelona 2005.

19. Molodecky N. Increasing incidente y prevalence of the inflamatory bowel diseases with time, base done systematic review. Gastroenterolpgy.2012;142(1):46-54.

20. Vieira SM, Pagovich OE. Diet, microbiota and autoinmune diseases. Lupus. 2014; 23(6):518-26.

21. Müller H, De Toledo FW. Fasting followed by vegetarian diet in patients with rheumatoid artritis: a systematic review. Scand J Rheumatol. 2001;30(1):1-10.

22. Chantret N, Salse J, Molecular basis of evolutionary events that shaped the hardness locus in diploide and polyploid wheat species (Triticum and Aegilops) Plant Cell. 2005 Apr 17(4):103345.

23. Seignalet J. Diet, fasting and rheumatoid artritis. Lancet 1992 4;339(8784):68-9.

24. Seignalet J. La alimentación, la tercera medicina: la dieta revolucionaria que ha curado a miles de personas. Barcelona: RBA; 2013. 
25. Häfstrom et al. A vegan diet free of gluten improves the signs and symptoms of rheumatoid arthritis: the effects on arthritis correlate with a reduction in antibodies to food antigens. Rheumatology 2000. 40(10):1175-79.

26. Lidén M.Cow's milk protein sensitivity assessed by the mucosal patch technique is related to irritable bowel syndrome in patients with primary Sjögren's syndrome.Clin Exp Allergy. 2008;38(6):929-35.

27.Ratner D. Milk protein-free diet for nonseasonal asthma and migraine in lactase-deficient patients.Isr J Med Sci. 1983 19(9): 806-9.

28. Friedman M. Bilogical effects of Maillard Browning products taht may affect acrylamide safety in food.effects of Maillard products. Adv Exp MEd Biol. 2005;561:135-156.

29. Zhang Y. Formation and reduction of acrylamide in Maillard reaction: a review base don the curent state of knowledge.Crit Rev Sci Nutr. 2007;47(5)521-42.

30. Verger $P$, Chambolle M. Estimation of the distribution of the maximum theoretical intake for ten additives in France. Food Addit Contam 1998;15(7):759-66.

31. Hercberg S, Preziosi P. Dietary intake in a representative sanmple of the population of Val-de Marne II Suply of macronutrients. Rev Epidemiol Sante Publique 1991;39(3):233-44

32. Hercberg S, Preziosi P. Dietary intake of a representative sample of the population of Val-de Marne III. Mineral and vitamine intake. Rev Epidemiol Sante Publique. 1991;39(3):245-61.

33. Maiden L, Thjodleifsssen B. Long-term effects of non steroidal anti-inflammatory drugs and ciclooxigenase-2 selective agents on the small bowel: a cross sectional capsule enteroscopy study. Clin Gastroenterol Hepatol. 2007;5 (9):1040-5.

34. Brzozowsky T, Konturek P. Phisiological mediators in non steroidal anti-inflammatory drugs (NSAID-s) induced impairment of gastic mucosal defense and adaptation. Focus on nitric oxide and lioxins. J Ohisiol Pharmacol 2008; 59 suppl 2:89-102.

\section{Opina sobre este artículo:}

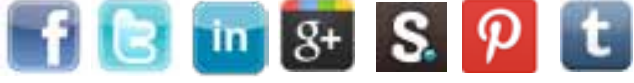

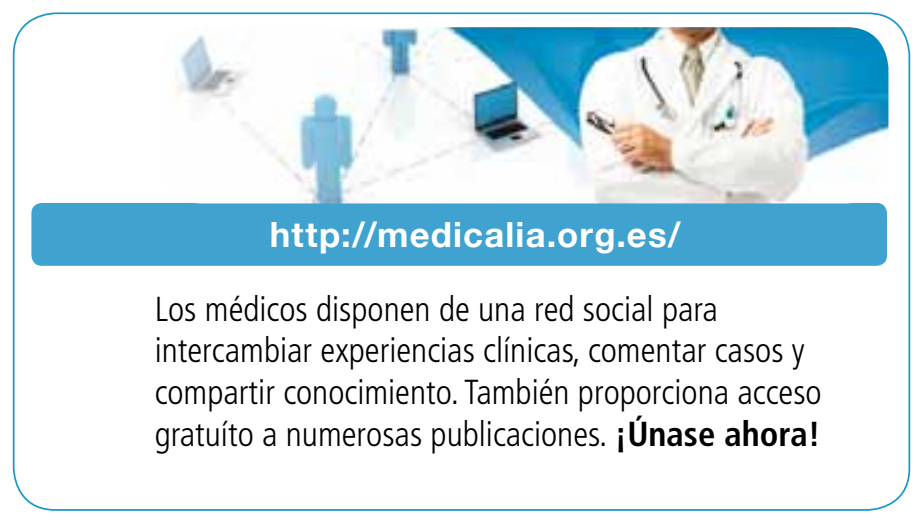

\section{Publish with iMedPub}

http://www.imed.pub

Acta Reumatológica es una revista que tiene por fin la difusión de estudios clínicos relacionados con aspectos prácticos del diagnóstico, tratamiento y seguimiento de pacientes con patología reumatológica, de estudios epidemiológicos relacionados con patología inflamatoria y musculoesquelética de presentación común o infrecuente en la práctica clínica tanto en población adulta como pediátrica, de casos clínicos de patología poco habitual o de presentaciones inhabituales de patología frecuente, de imágenes didácticas e ilustrativas en reumatología y del estado actual e innovación en la formación especializada en reumatología. 